\title{
THEORY OF HEAT CAPACITY OF LIQUID HELIUM-4 FOR TEMPERATURES ABOVE THE CRITICAL POINT
}

\author{
I.O. VAKARCHUK, V.S. PASTUKHOV, R.O. PRYTULA
}

PACS 67.25.k, 67.25.bd,

Ivan Franko National University of L'viv

(c) 2012

(12, Drahomanov Str., Lviv 79005, Ukraine; e-mail: volodyapastukhov@gmail. com)

\begin{abstract}
We analyze numerically the behavior of the heat capacity of liquid ${ }^{4} \mathrm{He}$ for the entire temperature range with the corresponding formula for the internal energy obtained in Ref. [I.O. Vakarchuk, R.O. Prytula, A.A. Rovenchak, J. Phys. Stud. 11, 259 (2007)] combined with a simple calculation of the effective mass of interacting Bose particles. The results agree quite well with experimental data.
\end{abstract}

\section{Introduction}

Notwithstanding a great number of papers (starting from Refs. [1,2]) concerned with the microscopic study of Bose system's properties, a good description of the heat capacity of liquid helium-4 in the whole temperature range has not yet been given. The first attempts were made in [3], where it was shown in the first order of perturbation theory for the free energy of a non-ideal Bose system that the presence of an interaction does change the order of the phase transition. In $[4,5]$, the thermodynamic functions of liquid helium-4 at all temperatures were obtained, by using the two-time temperature Green's function formalism. A good agreement of the specific heat at low temperatures with experimental data was obtained, and the temperature of the phase transition was calculated as $T_{c}=1.99 \mathrm{~K}$. The success of such an approach lies in the application of the experimentally measured structure factor of liquid helium-4 extrapolated to zero temperature [4] instead of the interparticle interaction potential. A good agreement of the heat capacity curve with relevant experimental data for the temperatures below the temperature of the $\lambda$ transition was obtained in Ref. [6], where the calculations were made, by using the quantum-statistical ap- proach based on the density matrix of a Bose liquid. At higher temperatures, the specific heat curve was shifted upward almost in a parallel way.

In Ref. [7], the density matrix formalism with the functional optimization of the Jastrow wave function parameters was used to describe the properties of liquid helium. The results for the internal energy agree well with experimental data for the temperatures below the critical one. It was shown that, by considering only the dynamic two-particle correlations, one can obtain the critical temperature to be $3.4 \mathrm{~K}$. Thus, to attain a good agreement with experiments, one needs to take higherorder approximations into account, which are specifically related to the concept of effective mass of a helium atom in the liquid.

In recent years, much attention has been paid to the study of atom's effective mass in liquid helium, because a part of the interaction could be taken into account in this way accordingly to Feynman's idea [8]. However, there is no satisfactory formula for the effective mass of a helium atom in the liquid at arbitrary temperatures. Various scholars were mostly concerned with the value of effective mass at $T \rightarrow 0$. Isihara and Samulski [9] have used the value of $m^{*} / m=1.71$ to agree the theoretically calculated sound branch of the excitation spectrum with the corresponding experimental data. In Ref. [10], the effective mass $m^{*} / m=1.70$ was obtained on the basis of measurements of the liquid helium- 4 structure factor. In Ref. [11], the interatomic potential was preserved as the input information, but, in part, the contribution of higher correlations was "transferred" to the kinetic energy term. In this way, the mass of particles was renormalized, which is somehow in correlation with the approach of Ref. [8]. As a result of such a renormalization, the value of $m^{*} / m=1.58$ was obtained using 
Green's function method. It was shown in Refs. [12] that the above-mentioned mass renormalization leads to the expressions obtained for the effective mass of a ${ }^{3} \mathrm{He}$ impurity atom in liquid ${ }^{4} \mathrm{He}$, but with the replacement of the "pure" ${ }^{3} \mathrm{He}$ atom mass by the ${ }^{4} \mathrm{He}$ atom mass.

The aim of this paper is to calculate the heat capacity of liquid helium above the phase transition temperature. The formula for the internal energy of a Bose liquid, which was obtained in Ref. [6] with the help of the method proposed in Ref. [13] (where the effective mass is a free parameter of the theory), forms the basis of these calculations. In what follows, we will calculate, step by step, the quasi-particle spectrum of the Bose system at the temperatures higher than the critical one and the effective mass with the help of thermodynamic perturbation theory and numerically analyze the behavior of the heat capacity.

\section{Perturbation Theory for the Grand Canonical Potential at $T>T_{c}$}

Consider a collection of $N$ spinless particles embedded into a volume $V$. The Hamiltonian of the system, which involves only a pair interaction between particles, may be written using the secondary quantization language as

$H=H_{0}+\Phi$

$H_{0}=\sum_{\mathbf{p}}\left(\varepsilon_{p}-\mu\right) a_{\mathbf{p}}^{+} a_{\mathbf{p}}$

$\Phi=\frac{N^{2}}{2 V} \nu(0)+\frac{1}{2} \sum_{\mathbf{k} \neq 0} \nu(k) \rho_{\mathbf{k}} \rho_{-\mathbf{k}}-\frac{N}{2 V} \sum_{\mathbf{k} \neq 0} \nu(k)$.

The creation $a_{\mathbf{p}}^{+}$and destruction $a_{\mathbf{p}}$ operators of a particle with momentum $\hbar \mathbf{p}$ satisfy the usual bosonic commutation relations. The notations $\nu(k)$ for the Fourier transform of the potential and $\varepsilon_{p}=\hbar^{2} p^{2} / 2 m$ for the free-particle spectrum are introduced. It is more convenient to work in the grand canonical ensemble. That is why we introduced the chemical potential $\mu$ and the fugacity $z=e^{\beta \mu}(\beta=1 / T$, where $T$ is the temperature) of the system that can be found with the help of the equation

$\sum_{\mathbf{p}}\left\langle a_{\mathbf{p}}^{+} a_{\mathbf{p}}\right\rangle=N$

Using the secondary quantization formalism, it is easy to write down the Fourier transform of the particle density fluctuation operator:

$\rho_{\mathbf{k}}=\frac{1}{\sqrt{V}} \sum_{\mathbf{p}} a_{\mathbf{p}}^{+} a_{\mathbf{p}+\mathbf{k}}, \quad(\mathbf{k} \neq 0)$.

Further, our task is to calculate the partition function of a many-boson system above the phase transition temperature. Of course, the most interesting features of these calculations occur in the region in a close vicinity of the temperature of the Bose condensation. As for the statistical operator, let us pass to the interaction representation and write down the partition function in the following way:

$Z=Z_{0} \exp \left\{-\beta \frac{N^{2}}{2 V} \nu(0)+\beta \frac{N}{2 V} \sum_{\mathbf{k} \neq 0} \nu(k)\right\} \times$

$\times\left\langle T_{\tau} \exp \left\{-\frac{1}{2} \int_{0}^{\beta} d \tau \sum_{\mathbf{k} \neq 0} \nu(k) \rho_{\mathbf{k}}(\tau) \rho_{-\mathbf{k}}(\tau)\right\}\right\rangle_{0}$.

Here, the quantity

$\rho_{\mathbf{k}}(\tau)=e^{\tau H_{0}} \rho_{\mathbf{k}} e^{-\tau H_{0}}$,

and the braces stand for the statistical averaging with the Hamiltonian $H_{0}$. The first multiplier $Z_{0}$ is the partition function of the ideal Bose gas. The second and third ones take completely the inter-particle interaction into account.

Next, we rewrite, with the help of the HubbardStratonovich transformation, the $T$-exponent in terms of the functional integral and average it over the states of the ideal Bose gas:

$Z / Z_{0}=\exp \left\{-\beta \frac{N^{2}}{2 V} \nu(0)+\beta \frac{N}{2 V} \sum_{\mathbf{k} \neq 0} \nu(k)\right\} \times$

$\times \int D \varphi \exp \left\{-\frac{1}{2} \sum_{q}(1+\nu(k) \Pi(q)) \varphi_{q} \varphi_{-q}+\right.$

$+\sum_{l \geq 3} \frac{(-i)^{l}}{l !(V \beta)^{l / 2-1}} \sum_{q_{1}} \ldots \sum_{q_{l}} \sqrt{\nu\left(k_{1}\right)} \ldots \sqrt{\nu\left(k_{l}\right)} \times$

$\left.\times \Pi_{l}\left(q_{1}, \ldots, q_{l}\right) \varphi_{q_{1}} \ldots \varphi_{q_{l}}\right\}$ 
Here, we use the notation $q=\left(\omega_{n}, \mathbf{k}\right), \omega_{n}=2 \pi n T(n=$ $0 ; \pm 1 ; \pm 2 ; \ldots)$ is the Matsubara frequency, and

$\sum_{q}=\sum_{\omega_{n}} \sum_{\mathbf{k} \neq 0}$.

The symbol $\int D \varphi$ denotes the integration over the real and imaginary parts of the $\varphi_{q}$ variables from the halfspace of all possible values of $q$ due to the symmetry $\varphi_{q}^{*}=\varphi_{-q}$. The polarization operator

$$
\begin{aligned}
& \Pi(q)=\Pi\left(\omega_{n}, k\right)=\frac{1}{V \beta} \sum_{q^{\prime}} G_{0}\left(q^{\prime}\right) G_{0}\left(q^{\prime}+q\right)= \\
& =\frac{1}{V} \sum_{\mathbf{k}^{\prime}} \frac{n_{\left|\mathbf{k}^{\prime}+\mathbf{k}\right|}-n_{k^{\prime}}}{i \omega_{n}-\varepsilon_{\left|\mathbf{k}^{\prime}+\mathbf{k}\right|}+\varepsilon_{k^{\prime}}} .
\end{aligned}
$$

Here and thereafter, $n_{k}$ is the filling factor of the ideal Bose gas. We also introduced notations for the symmetric functions:

$\Pi_{l}\left(q_{1}, \ldots, q_{l}\right)=\frac{1}{V \beta l} \sum_{q}\left\{G_{0}(q) G_{0}\left(q+q_{1}\right) \times\right.$

$\times \ldots G_{0}\left(q+q_{1}+\ldots+q_{l-1}\right)+$ permutations $\}$.

The one-particle Green's function of non-interacting bosons is

$G_{0}(q)=\frac{1}{i \omega_{n}-\varepsilon_{k}+\mu}$.

Representation (5), where the partition function is written in terms of functional integrals, was used successfully in the theory of Fermi systems with the Coulombic interaction in Ref. [14]. The fact that we recover Random Phase Approximation (RPA) correctly in the calculation of the functional integral in the Gaussian approximation is a great advantage of our method. This approximation in the case of interacting fermions generalizes the wellknown result of Gell-Mann-Brueckner for a high-density electron gas at finite temperatures. The non-Gaussian part can be taken into account approximately by means of perturbation theory.

The thermodynamic potential up to the first order of perturbation theory (in the two-sum approximation over the "4-vector") is

$\Omega=\Omega_{0}+\Omega_{1}+\Omega_{2}$,

where

$\Omega_{0}=T \sum_{\mathbf{p}} \ln \left(1-z e^{-\beta \varepsilon_{p}}\right)$ is the ideal gas contribution, and

$$
\Omega_{1}=\frac{N^{2}}{2 V} \nu(0)-\frac{N}{2 V} \sum_{\mathbf{k} \neq 0} \nu(k)+
$$

$+\frac{1}{2 \beta} \sum_{q} \ln |1+\nu(k) \Pi(q)|$

is the well-known [15] RPA-part of the grand canonical potential. Formula (10) was analyzed in detail only for Fermi systems. As far as we know, the properties of liquid helium-4 for the entire temperature range in RPA never were studied. As we work in the grand canonical ensemble, we take the average number of particles $N$ as a function of the chemical potential $\mu$.

The one-loop contribution to the thermodynamic potential is

$$
\begin{aligned}
& \Omega_{2}=\frac{1}{2 \cdot 3 ! V \beta^{2}} \sum_{\substack{q_{1} \\
q_{1}+q_{2}+q_{3}=0}} \sum_{q_{2}} \sum_{3} \Pi_{3}^{2}\left(q_{1}, q_{2}, q_{3}\right) \times \\
& \times \frac{\nu\left(k_{1}\right)}{1+\nu\left(k_{1}\right) \Pi\left(q_{1}\right)} \frac{\nu\left(k_{2}\right)}{1+\nu\left(k_{2}\right) \Pi\left(q_{2}\right)} \frac{\nu\left(k_{3}\right)}{1+\nu\left(k_{3}\right) \Pi\left(q_{3}\right)}-
\end{aligned}
$$

$-\frac{1}{8 V \beta^{2}} \sum_{q_{1}} \sum_{q_{2}} \Pi_{4}\left(q_{1},-q_{1}, q_{2},-q_{2}\right) \times$

$$
\times \frac{\nu\left(k_{1}\right)}{1+\nu\left(k_{1}\right) \Pi\left(q_{1}\right)} \frac{\nu\left(k_{2}\right)}{1+\nu\left(k_{2}\right) \Pi\left(q_{2}\right)} .
$$

The structure of expressions (10) and (11) clearly shows that they are "in correlation" with the formulae obtained in Ref. [6], where an entirely different method of calculations was used.

\section{Renormalization of the One-Particle Spectrum}

It is clear that the basis of further calculations is fully determined by the renormalization of the quasi-particle spectrum. For our analysis, we use RPA. Notwithstanding the simplicity of this approximation, it "catches" certain important features of the behavior of the system. It is not surprising, because RPA effectively sums up an infinite set of terms of perturbation theory divergent near the phase transition point.

At first, let us use the thermodynamic equality $-\partial \Omega / \partial \mu=N$ to find the average number of particles in 
the system. The explicit calculation of the corresponding derivative with the first two terms of Eq. (8) gives

$$
\begin{aligned}
& N=\sum_{\mathbf{p}}\left\{n_{p}-\frac{\partial n_{p}}{\partial \mu} \frac{N}{V} \nu(0)+\frac{\partial n_{p}}{\partial \mu} \frac{1}{2 V} \sum_{\mathbf{k} \neq 0} \nu(k)-\right. \\
& -\frac{\partial n_{p}}{\partial \mu} \frac{1}{2 V \beta} \sum_{q} \frac{\nu(k)}{1+\nu(k) \Pi(q)} \times \\
& \left.\times\left[\frac{1}{\varepsilon_{|\mathbf{k}-\mathbf{p}|}-\varepsilon_{p}-i \omega_{n}}+\text { c. c. }\right]\right\} .
\end{aligned}
$$

Let us construct the Bose filling factor with a new spectrum using the expression in braces. Making use of the equality $\partial n_{p} / \partial \mu=-\partial n_{p} / \partial \varepsilon_{p}$, we finally obtain the formula for the renormalized one-particle spectrum,

$\varepsilon_{p}^{*}=\varepsilon_{p}+\Delta \varepsilon_{p}, \quad \mu^{*}=\mu+\Delta \mu$,

where the correction to the quasi-particle spectrum is

$$
\begin{aligned}
& \Delta \varepsilon_{p}=\frac{1}{\beta V} \sum_{q} \frac{\nu(k)}{1+\nu(k) \Pi(q)}\left\{\frac{1}{\varepsilon_{|\mathbf{k}-\mathbf{p}|}-\varepsilon_{p}-i \omega_{n}}-\right. \\
& \left.-\frac{1}{\varepsilon_{k}-i \omega_{n}}\right\}
\end{aligned}
$$

and the correction to the chemical potential is

$$
\begin{aligned}
& \Delta \mu=-\frac{N}{V} \nu(0)+\frac{1}{2 V} \sum_{\mathbf{k} \neq 0} \nu(k)- \\
& -\frac{1}{\beta V} \sum_{q} \frac{\nu(k)}{1+\nu(k) \Pi(q)} \frac{\varepsilon_{k}}{\varepsilon_{k}^{2}+\omega_{n}^{2}} .
\end{aligned}
$$

It is easy to obtain the above-mentioned expression for the spectrum in a different way. To do this, one has to recall that variational derivative of the $\Omega$-potential with respect to $\varepsilon_{p}$ equals the renormalized one-particle filling factor $n_{p}^{*}$. After simple calculations, we obtain the formula

$$
\begin{aligned}
& n_{p}^{*}=n_{p}+\frac{N}{V} \nu(0) \frac{\partial n_{p}}{\partial \varepsilon_{p}}-\frac{1}{2 V} \sum_{\mathbf{k} \neq 0} \nu(k) \frac{\partial n_{p}}{\partial \varepsilon_{p}}+ \\
& +\frac{1}{2 V \beta} \sum_{q} \frac{\nu(k)}{1+\nu(k) \Pi(q)} \times
\end{aligned}
$$

$\times\left\{\left[\frac{1}{\varepsilon_{|\mathbf{k}-\mathbf{p}|}-\varepsilon_{p}-i \omega_{n}}+\right.\right.$ c. c. $] \frac{\partial n_{p}}{\partial \varepsilon_{p}}+$

$+\left[\frac{n_{p}-n_{|\mathbf{k}-\mathbf{p}|}}{\left(\varepsilon_{|\mathbf{k}-\mathbf{p}|}-\varepsilon_{p}-i \omega_{n}\right)^{2}}+\right.$ c.c. $\left.]\right\}$.

It is easy to argue by making summation over the wavevector $\mathbf{p}$ of the left- and right-hand sides of the previous equality that the second term in braces vanishes. So, after the summation of expression (16), we arrive at equality (12), and, thus, we get the same expression (14) for the correction to a one-particle spectrum again. It is interesting to note that the calculation of the variation derivative $\delta \Omega / \delta n_{p}$ in RPA gives the same result. Finally, Eq. (14) coincides with the result derived in Ref. [16], where calculations were made in terms of the temperature Green's function technique.

Let us analyze expression (14). First, the potential problems with the integration over the wave-vector may occur only in the critical region and at the zero frequency $\omega_{n}$. That is why we write down this term apart and immediately set apart the Hartree-Fock-like term

$$
\begin{aligned}
& \Delta \varepsilon_{p}=\frac{1}{\beta V} \sum_{\mathbf{k} \neq 0} \frac{\nu(k)}{1+\nu(k) \Pi(0, k)}\left[\frac{1}{\varepsilon_{|\mathbf{k}-\mathbf{p}|}-\varepsilon_{p}}-\frac{1}{\varepsilon_{k}}\right]+ \\
& +\frac{1}{V} \sum_{\mathbf{k} \neq 0} \nu(k)\left\{n\left(\beta \varepsilon_{|\mathbf{k}-\mathbf{p}|}-\beta \varepsilon_{p}\right)-n\left(\beta \varepsilon_{k}\right)-\right. \\
& \left.-\frac{1}{\beta\left[\varepsilon_{|\mathbf{k}-\mathbf{p}|}-\varepsilon_{p}\right]}+\frac{1}{\beta \varepsilon_{k}}\right\}-\frac{1}{\beta V} \sum_{q \neq 0} \frac{\nu^{2}(k) \Pi\left(\omega_{n}, k\right)}{1+\nu(k) \Pi\left(\omega_{n}, k\right)} \times \\
& \times\left[\frac{1}{\varepsilon_{|\mathbf{k}-\mathbf{p}|}-\varepsilon_{p}-i \omega_{n}}-\frac{1}{\varepsilon_{k}-i \omega_{n}}\right]
\end{aligned}
$$

where $n(x)=1 /\left(e^{x}-1\right)$, and $\rho=N / V$ is the equilibrium density of the system. Second, to go further, we have to investigate the properties of the polarization operator (6)

$$
\begin{aligned}
& \Pi\left(\omega_{n}, k\right)=\frac{\beta k_{0}^{3}}{(2 \pi)^{2}} \frac{k_{0}}{2 k} \int_{0}^{\infty} d x \frac{x}{z^{-1} e^{x^{2}-1}} \times \\
& \times\left\{\ln \left|\frac{\left(k / k_{0}\right)^{2}+2 x k / k_{0}-i u_{n}}{\left(k / k_{0}\right)^{2}-2 x k / k_{0}-i u_{n}}\right|+\text { c. c. }\right\} .
\end{aligned}
$$


For convenience, the following notations are used: $k_{0}=$ $\sqrt{2 m T} / \hbar, u_{n}=2 \pi n$. We are interested in a long wavelength behavior of the polarization operator. To find the leading order asymptote of $\Pi\left(\omega_{n}, k\right)$ at the zero frequency at the critical point, it is sufficient to replace the Bose filing factor $1 /\left(e^{x^{2}}-1\right)$ in integral (18) by $1 / x^{2}$. Then, after a simple calculation, we get $\Pi(0, k \rightarrow 0)=$ $\beta k_{0}^{4} /(8 k)$. For higher temperatures $(\mu \neq 0)$, we have

$$
\begin{aligned}
& \Pi(0, k)=\beta \rho\left\{\frac{g_{1 / 2}\left(e^{\beta \mu}\right)}{g_{3 / 2}\left(e^{\beta \mu}\right)}-\frac{1}{6} \frac{k^{2}}{k_{0}^{2}} \frac{g_{-1 / 2}\left(e^{\beta \mu}\right)}{g_{3 / 2}\left(e^{\beta \mu}\right)}\right\}+\ldots \\
& g_{l}\left(e^{y}\right)=\sum_{n \geq 1} \frac{e^{y n}}{n^{l}} .
\end{aligned}
$$

The dots stand for the terms higher than quadratic ones in the expansion in the wave-vector. It is easy to see from definition (6) that $\Pi\left(\omega_{n}, 0\right)=0$ for non-zero frequencies.

Now, let us consider the contribution to the oneparticle spectrum (14) from the zero frequency. Using the designation $\Delta \varepsilon_{p}^{u}$ for this term of the spectrum, it is easy to write down

$$
\begin{aligned}
& \Delta \varepsilon_{p}^{u}=\left(k_{0} / \pi\right)^{2} p \int_{0}^{\infty} d x \frac{\nu(2 x p)}{1+\rho \nu(2 x p) \Pi(0,2 x p)} \times \\
& \times\left[\frac{x}{2} \ln \left|\frac{x+1}{x-1}\right|-1\right] .
\end{aligned}
$$

For the self-consistency of our calculations, especially near the critical point, the chemical potential $\mu$ should be changed by $\mu^{*}$ on the right-hand side of Eq. (20) (the critical point is determined by the equation $\mu^{*}=0$, respectively). Admittedly, the ideal gas dispersion relation should be replaced by the exact one-particle spectrum, but the further analysis will not be influenced by this replacement qualitatively.

Let us consider the integral in Eq. (20) at small $p$ and assume, for definiteness, that the temperature is higher than the critical one. Then, substituting $\Pi(0,2 x p) \rightarrow$ $\Pi(0,0)$ and $\nu(2 x p) \rightarrow \nu(0)$, we obtain

$$
\Delta \varepsilon_{p}^{u}=\frac{k_{0}^{2}}{\pi^{2}} p \frac{\nu(0)}{1+\rho \nu(0) \Pi(0,0)} \int_{0}^{\infty} d x\left[\frac{x}{2} \ln \left|\frac{x+1}{x-1}\right|-1\right] .
$$

It turns out that this integral equals zero identically. Moreover, even after the substitution of $\Pi(0, k) \rightarrow(19)$, the integral in Eq. (20) equals zero too. Thus, it is shown, with a realistic restriction on the Fourier transform of the potential energy, i.e. in the absence of linear and quadratic terms in the expansion of $\nu(k)$ at small $k$, that $\Delta \varepsilon_{p}^{u}=o\left(p^{2}\right)$.

The situation is quite different in the critical region. Here, the leading-order asymptote is $\Delta \varepsilon_{p}^{u} \sim p^{2} \ln (p)$ (it is easy to show with regard for the properties of $\Pi(0, k) \sim 1 / k$ in this region), which obviously is a hint at the following behavior of the one-particle spectrum $\Delta \varepsilon_{p}^{u} \sim p^{2-\eta}(\eta \rightarrow 0)$ at the critical temperature. Clearly, one cannot obtain this result using a simple perturbative approach.

Hence, having separated the non-analytic problematic part of the spectrum (the second term in Eq. (17)), we can consider the "non-universal" one, i.e. the remainder of Eq. (17). Precisely this expression will determine the observed non-universal properties of the Bose liquid. The latter calculations are linked to the summation over the Matsubara frequencies in the last term of Eq. (17) and coincide with those in Ref. [16]. That is why we do not dwell on the details of these calculations. Now, the leading-order non-vanishing term of the quasi-particle dispersion relation is quadratic in the wave-vector. We recall that $\Delta \varepsilon_{p}^{u}=o\left(p^{2}\right)$, and, hence, its contribution is not significant. So, for simplification, we assume the spectrum to be a quadratic free-particle one, but with the normalized mass.

As we single out the "problematic" contribution to the quasi-particle spectrum, by expanding the second and third sums of Eq. (17) in series in $p$, we obtain that the one-particle spectrum reads

$\varepsilon_{p}^{*}=\Delta \varepsilon_{p}^{u}+\frac{\hbar^{2} p^{2}}{2 m^{*}}$.

The effective mass is

$m / m^{*}=1-\Delta(T)$,

where the quantity

$$
\begin{aligned}
& \Delta(T)=\frac{1}{3 N} \sum_{\mathbf{k} \neq 0} \frac{\left(\alpha_{k}-1\right)^{2}}{\alpha_{k}\left(\alpha_{k}+1\right)}+ \\
& +\frac{2}{3 N} \sum_{\mathbf{k} \neq 0}\left\{\frac{\alpha_{k}^{2}+3}{\alpha_{k}^{2}-1}\left[n\left(\beta \varepsilon_{k}\right)-1 / \beta \varepsilon_{k}\right]-\right. \\
& -\frac{3 \alpha_{k}^{2}+1}{\alpha_{k}\left(\alpha_{k}^{2}-1\right)}\left[n\left(\beta E_{k}\right)-1 / \beta E_{k}\right]+ \\
& \left.+2\left[1 / \beta \varepsilon_{k}-\beta \varepsilon_{k} n\left(\beta \varepsilon_{k}\right)\left[1+n\left(\beta \varepsilon_{k}\right)\right]\right]\right\} .
\end{aligned}
$$


Here, we use the following notations: $E_{k}=\alpha_{k} \varepsilon_{k}$ is the Bogoliubov spectrum, and $\alpha_{k}=\sqrt{1+2 \rho \nu(k) / \varepsilon_{k}}$. The effective mass in the low-temperature region is always larger than its "bare" one, which means that the renormalized temperature of the Bose condensation of interacting particles is always lower than the critical temperature of the ideal gas. This is the most important result of Eqs. (22) and (23). Carefully calculating the limit $\beta \rightarrow 0$ at low temperatures, it is easy to ascertain that the effective mass tends to the mass of particles. It is of importance that the temperature-independent part of formula (23) coincides with the effective mass derived in Ref. [12], where a different method was used in calculations.

At the end of this section, one more remark on the applicability of the formula for $\Delta(T)$ has to be made. The approximation of the exact spectrum of collective modes by the Bogoliubov spectrum, on the one hand, made it possible to obtain the analytical expression (23). On the other hand, it brought us beyond the limits of RPA. It is hard to assess the accuracy of such a trick, but it can be justified, only by considering that the Fourier transform of the two-particle potential is a rapidly decreasing function. Then the main contribution to the integral over the wave-vector comes from the lower limit of integration, where the Bogoliubov spectrum completely coincides with the exact one.

\section{Internal Energy and Heat Capacity}

The expression for the grand canonical potential derived in Section 2 is applicable only for the temperatures higher than the critical one. To describe the $\lambda$-transition phenomenon, in particular thermodynamic functions, let us use an approach based on the density matrix of the Bose liquid [13]. Using this approach, the dependence of the internal energy of the Bose liquid on the effective mass of a helium- 4 atom was found in the approximation of pair-particle correlations in Ref. [6]. In the case of $m^{*} / m=1$, a good agreement of the heat capacity with experimental data in the region below the critical point was obtained in Ref. [6]. But above the phase transition temperature, the heat capacity curve was shifted upward. Hence, the calculation of the heat capacity for the case of $m^{*} / m \neq 1$ is an interesting problem.

We take the expression for the internal energy in the approximation of pair-particle correlations from Ref. [6]:

$$
E=N \frac{m c^{2}}{2}+\sum_{\mathbf{k} \neq 0} \frac{\hbar^{2} k^{2}}{2 m} \frac{1}{z_{0}^{-1} e^{\beta \varepsilon_{k}^{*}-1}}+
$$

$$
\begin{aligned}
& +\frac{1}{2} \frac{m^{*}}{m} \sum_{\mathbf{k} \neq 0} \frac{\lambda_{k}}{1+\lambda_{k} S_{0}(k)} \frac{\partial S_{0}(k)}{\partial \beta}+ \\
& +\frac{1}{4} \sum_{\mathbf{k} \neq 0} \frac{\hbar^{2} k^{2}}{2 m}\left(\lambda_{k}^{2}+\alpha_{k}^{2}-1\right) S(k)+ \\
& +\frac{1}{2} \sum_{\mathbf{k} \neq 0} \frac{\hbar^{2} k^{2}}{2 m}\left[\frac{\alpha_{k}}{\sinh \left(\beta E_{k}\right)}-\frac{1}{\sinh \left(\beta \varepsilon_{k}^{*}\right)}\right]+ \\
& +\frac{1}{16} \sum_{\mathbf{k} \neq 0} \frac{\hbar^{2} k^{2}}{2 m}\left(1-\frac{1}{\alpha_{k}^{2}}\right)\left(\alpha_{k}-\frac{1}{\alpha_{k}}-4 \alpha_{k}^{2}\right) .
\end{aligned}
$$

Here, the following notations are introduced:

$$
S_{0}(k)=1+2 \frac{n_{0}}{N} n_{k}^{*}+\frac{1}{N} \sum_{\substack{\mathbf{p} \neq 0 \\ \mathbf{p}+\mathbf{k} \neq 0}} n_{p}^{*} n_{|\mathbf{p}+\mathbf{k}|}^{*}
$$

is the structure factor of the ideal Bose gas with a renormalized mass. The quantity $n_{0}$ is the average number of particles of the ideal Bose gas with zero momentum, and $z_{0}$ is its fugacity.

The quantity

$$
S(k)=\frac{S_{0}(k)}{1+\lambda_{k} S_{0}(k)}
$$

is the pair structure factor of the Bose liquid, and

$\lambda_{k}=\alpha_{k} \tanh \left(\beta E_{k} / 2\right)-\tanh \left(\beta \varepsilon_{k}^{*} / 2\right)$.

Let us make remark about formulae (13) and (24). These formulas were obtained with the use of different approximate approaches; that is why the chemical potential calculated from Eq. (24) $\widetilde{\mu}=(\partial E / \partial N)_{T}$ does not coincide with Eq. (13).

Obviously, if we turn off the interparticle interaction $\alpha_{k}=1, m^{*}=m$, and take into account that the sound velocity in the ideal Bose gas at $T=0$ equals zero, $c=0$ (there are no zero density fluctuations in the Bose system), and $\lambda_{k}=0$ (27), we obtain the well-known formula for the energy of the ideal Bose gas

$E=\sum_{\mathbf{k} \neq 0} \frac{\hbar^{2} k^{2}}{2 m} \frac{1}{z_{0}^{-1} e^{\beta \hbar^{2} k^{2} / 2 m}-1}$.

Let us analyze the total energy (24) in the lowtemperature region, where it coincides with the formulae obtained in Ref. [13,17]. At low temperatures $T \rightarrow 0$, 


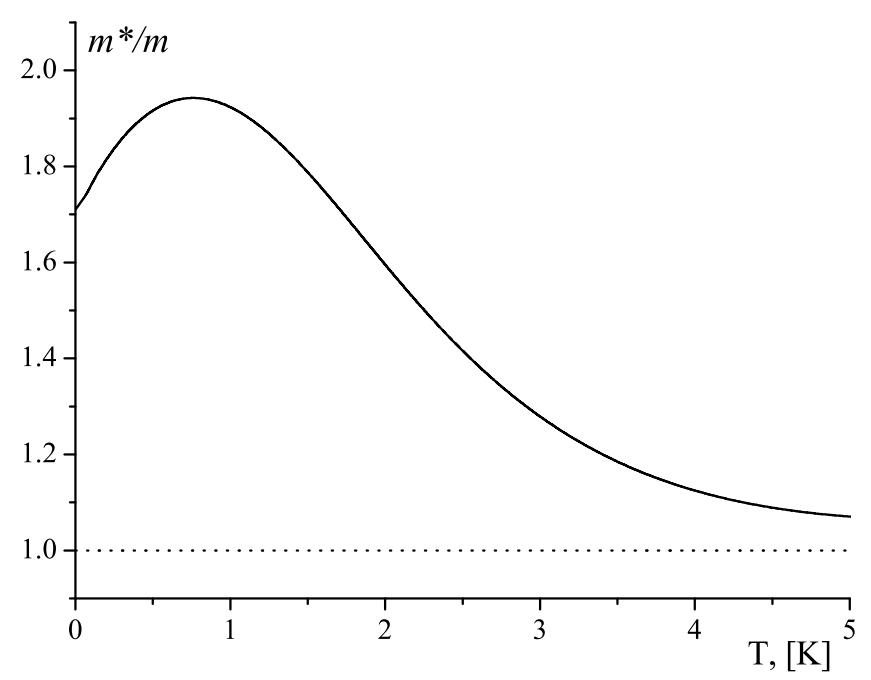

Fig. 1. Temperature dependence of the fraction $m^{*} / m$

when only small values of the wave-vector $\mathbf{k}$ are important in the expression for the spectrum $E_{k}$, we obtain

$E=E_{0}+\sum_{\mathbf{k} \neq 0} \frac{E_{k}}{e^{\beta E_{k}}-1}$,

where the ground-state energy is

$E_{0}=N \frac{m c^{2}}{2}-\frac{1}{4} \sum_{\mathbf{k} \neq 0} \frac{\hbar^{2} k^{2}}{2 m}\left(\alpha_{k}-1\right)^{2}+$

$+\frac{1}{16} \sum_{\mathbf{k} \neq 0} \frac{\hbar^{2} k^{2}}{2 m} \frac{1}{\alpha_{k}}\left(\alpha_{k}-\frac{1}{\alpha_{k}}\right)^{2}$,

and, after explicit calculations,

$E=E_{0}+\frac{V \pi^{2}}{30(\hbar c)^{3}} T^{4}, \quad C_{V}=V \frac{2 \pi^{2}}{15(\hbar c)^{3}} T^{3}$.

Exactly the same temperature dependence of the heat capacity of liquid ${ }^{4} \mathrm{He}$ at $T \rightarrow 0$ is observed. Since we obtained the correct behavior of the heat capacity at low temperatures, by using the energy of the Bose liquid (24), we expect to derive the correct behavior of the heat capacity for the entire temperature range.

\section{Numerical Results}

Our numerical calculations are carried out at the equilibrium density of liquid helium $\rho=0.02185 \AA^{-3}$, mass of particles $m=4.0026 \mathrm{u}$, sound velocity $c=238.2 \mathrm{~m} / \mathrm{s}$ in the limit of $T \rightarrow 0$ [18], and at the critical temperature of the ideal gas $T_{c}=3.138 \mathrm{~K}$. We use the liquid structure factor extrapolated to $T=0$ [4] as the output information, instead of the interparticle potential, i.e.

$\alpha_{k}=\frac{1}{S^{\exp (k)}}$,

where $S^{\exp }(k)$ is the experimentally measured structure factor at $T=0$.

It is logical to start the calculations with the formula for the renormalized one-particle spectrum and, thus, with the formula for the effective mass of particles. Despite the complexity of the last sum over the wave-vector in Eq. (23), the main contribution to the effective mass arises from the temperature-independent part. In Fig. 1 , the dependence of the dimensionless effective mass $m^{*} / m$ on the temperature is presented. Formally, we extrapolated the curve of the effective mass in the condensate region, where it becomes obviously a parameter of the theory. It is important that, at the zero temperature, $m^{*}$ coincides with the effective mass of an impurity atom in the Bose liquid [12].

The first three terms of the low-temperature expansion are $\left(T \ll m c^{2}, T \ll \hbar^{2} \rho^{2 / 3} / m\right)$, and

$\Delta(T \rightarrow 0)=\Delta_{0}+\Delta_{1} T-\Delta_{3 / 2} T^{3 / 2}+O\left(T^{5 / 2}\right)$,

( $T$ in Kelvins)

where $\Delta$-coefficients are

$\Delta_{0}=\frac{1}{3 N} \sum_{\mathbf{k} \neq 0} \frac{\left(\alpha_{k}-1\right)^{2}}{\alpha_{k}\left(\alpha_{k}+1\right)}=0.41$,

$\Delta_{1}=\frac{2}{3 N} \sum_{\mathbf{k} \neq 0} \frac{\alpha_{k}^{2}-1}{\alpha_{k}^{2} \varepsilon_{k}}=0.31$

$\Delta_{3 / 2}=\frac{4}{3} \zeta(3 / 2)\left(\frac{m}{2 \pi \hbar^{2}}\right)^{3 / 2} / \rho=0.24$

Then the effective mass equals approximately

$m^{*}=m /\left(0.59-0.31 T+0.24 T^{3 / 2}\right)$.

This formula reproduces the curve in Fig. 1 quite well up to the critical temperature.

We now are in position to calculate the renormalized temperature of the Bose condensation. We can find $T_{c}$ using the condition $\sum_{\mathbf{p}} n_{p}^{*}=N$ at the zero value of the renormalized chemical potential $\mu^{*}$. A simple calculation gives $T_{c}=1.94 \mathrm{~K}$ that agrees quite well with 
experimental measurements of the temperature of the $\lambda$-transition $T_{c}^{\exp }=2.17 \mathrm{~K}$ despite the simplicity of the approximations.

Let us pass on to the heat capacity calculation:

$C_{V}=\left(\frac{\partial E}{\partial T}\right)_{V}$

We calculate the heat capacity using the difference method and build the plot of its dependence on $T / T_{c}$.

A comparison of different heat capacity curves is depicted in Fig. 2. As is seen from the comparison of the calculated curve 1 with the experimental one, the agreement is quite good at low temperatures $\left(0<T / T_{c}<1\right)$. At the temperatures $T / T_{c}>1$, the inconsistency occurs: the behavior of the calculated heat capacity is very similar to the behavior of the experimental curve, but shifted upward almost in a parallel way. This inconsistency is related to the fact that the three- and four-particle correlations should be taken into account for the quantitative description. The contribution of the three- and four-particle correlations, as is shown in Refs. [17, 19], improves significantly the ground-state results and gives a fairly good agreement at $T \rightarrow 0$.

Further, let us calculate the heat capacity of liquid helium-4 with regard for the effective mass of a helium atom in the liquid (curve 2). At low temperatures, the heat capacity practically coincides with curve 1 , which agrees well with experimental data. This shows the weak dependence of the heat capacity on the effective mass below the phase transition temperature. As is seen from Fig. 2, the calculated curve 2 (unlike curve 1) agrees quite well with the experimental one. It is related to the fact that, by using the effective mass, we partially take the contribution from the three- and four-particle correlations into account. It is not surprising that, in a close vicinity of the Bose condensation point, the theoretically calculated heat capacity deviates most significantly from the experimental curve. It is solely related to the inconsistency of our description near the critical point because the non-analytical part (20) of the one-particle spectrum, which makes a significant contribution to the thermodynamic functions at $T \rightarrow T_{c}$, is disregarded in our approach. One has to use renormalization group methods [24-26] for the correct description of the heat capacity in this temperature region.

\section{Conclusions}

We have succeeded in deriving quite well an agreement of the heat capacity curve for liquid helium with experi-

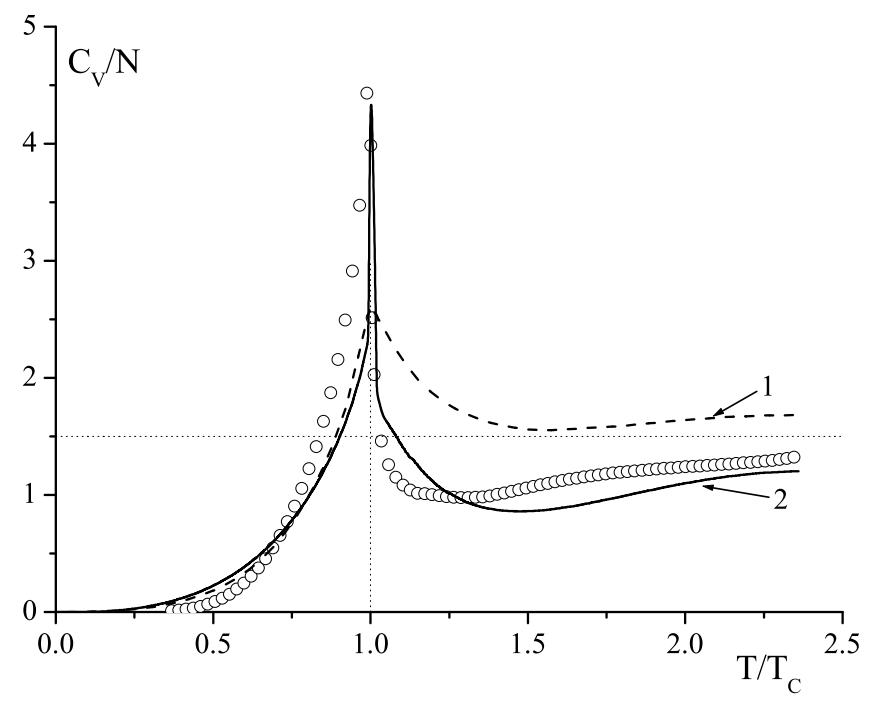

Fig. 2. Heat capacity of liquid helium-4. Curve 1 is the calculated heat capacity in the pair-correlation approximation (24) [6]; curve 2 is the heat capacity in the pair-correlation approximation (24) with regard for the effective mass of a helium atom in the liquid (22). The circles show experimental data from Refs. [20-22]

mental data practically for all temperatures. The application of the thermodynamic functions of the Bose liquid obtained with the help of the Hamiltonian averaging combined with a one-particle spectrum derivation were the key moments of calculations. Notwithstanding the simplicity of the spectrum calculation, we obtained quite interesting results. In particular, we can decompose the part of the quasiparticle spectrum that is responsible for the non-analyticity at the Bose condensation point and show that this term of the spectrum has no effect on the physical observables in the undercritical temperature region. So, the attempt is made to justify microscopically the idea that the $\lambda$-transition in a real quantum liquid is very similar to the Bose-Einstein condensation phenomenon in the ideal gas "slightly" deformed by the interaction between the particles (keeping in mind the non-universal properties of the system).

The calculation has found that the long wave-length limit of the "non-universal" part of the one-particle spectrum is quadratic in the wave-vector, i.e. very similar to the dispersion relation of the ideal gas, but with a new mass. In the general case, it is shown that this new mass at low temperatures is always greater than the mass of particles, and thus, the presence of the interaction always lowers, at least in our approximation, the critical temperature.

Another feature of the developed theory, perhaps a bit unexpected, is that even this simple temperature de- 
pendence of the effective mass improves the behavior of the heat capacity curve in the undercritical region and does not affect it in the condensate phase. Hence, the quantum-statistical approach based on the density matrix is suitable to describe the thermodynamic properties of such a strongly-interacting Bose liquid as helium-4 not only in the limits of low and high temperatures, but in the entire temperature range.

1. N.N. Bogoliubov, J. Phys. (USSR) 11, 23 (1947).

2. N.N. Bogoliubov and D.N. Zubarev, Sov. Phys.-JETP 1, 83 (1955).

3. R. Brout, Phase Transitions (Benjamin, New York, 1965).

4. I.O. Vakarchuk, V.V. Babin, and A.A. Rovenchak, J. Phys. Stud. 4, 16 (2000).

5. I.O. Vakarchuk and A.A. Rovenchak, J. Phys. Stud. 5, 126 (2001); Condens. Matter. Phys. 4, 431 (2001).

6. I.O. Vakarchuk, R.O. Prytula, and A.A. Rovenchak, J. Phys. Stud. 11, 259 (2007).

7. T. Lindenau, M.L. Ristig, J.W. Clark, and K.A. Gernoth, J. Low Temp. Phys. 129, 143 (2002).

8. R.P. Feynman, Phys. Rev. 91, 1291 (1953).

9. A. Isihara and T. Samulski, Phys. Rev. B 16, 1969 (1977).

10. I.O. Vakarchuk, Lviv Univer. Bull. 26, 29 (1993).

11. A.A. Rovenchak, Fiz. Nizk. Temp. 29, 145 (2003).

12. I. O. Vakarchuk, J. Phys. Stud. 1, 25 (1996); J. Phys. Stud. 1, 156 (1997).

13. I.O. Vakarchuk, J. Phys. Stud. 8, 223 (2004).

14. M. Vavrukh and T. Krokhmalskii, Phys. Stat. Sol. 168, 519 (1991).

15. N.M. March, W.H. Young, S. Sampanthar, The ManyBody Problems in Quantum Mechanics (Cambridge University Press, Cambridge, 1967).
16. I.O. Vakarchuk and V.S. Pastukhov, J. Phys. Stud. 15, 3003 (2011).

17. I.A. Vakarchuk, Theor. Math. Phys. 82, 308 (1990).

18. R.J. Donnelly and C.F. Barenghi, J. Phys. Chem. Ref. Data. 27, 1217 (1998).

19. I.A. Vakarchuk and P.A. Glushak, Theor. Math. Phys. 75, 399 (1988).

20. V.D. Arp, Int. J. Thermophys. 26, 1477 (2005).

21. D.M. Ceperley, Rev. Mod. Phys. 67, 279 (1995).

22. V.D. Arp, R.D. McCarty, and D.G. Friend, Natl. Inst. Stand. Technol. Tech. Note 1334 (revised) (1998).

23. J.A. Lipa, J.A. Nissen, D.A. Stricker, D.R. Swanson, and T.C.P. Chui, Phys. Rev. B 68, 174518 (2003).

24. J. Kaupuzs, Eur. Phys. J. B 45, 459 (2005).

25. M. Campostrini, M. Hasenbusch, A. Pelissetto, and E. Vicari, Phys. Rev. B 74, 144506 (2006).

26. A.A. Pogorelov and I.M. Suslov, JETP Letters 86, 39 (2007).

Received 26.12.11

ТЕОРІЯ ТЕПЛОЄМНОСТІ РІДКОГО ГЕЛІЮ-4

ДЛЯ ТЕМПЕРАТУР ВИЩИХ КРИТИЧНОї

I.О. Вакарчук, В.С. Пастухов, Р.О. Притула

Р е $з$ ю м е

В статті, за допомогою виразу для внутрішньої енергії, отриманого в роботі [6], в поєднанні з простим розрахунком ефективної маси взаємодіючих бозе-частинок, чисельно проаналізовано поведінку теплоємності рідкого ${ }^{4}$ Не для всіх значень температури. Результати добре узгоджуються з експериментальними даними. 